\title{
Prognostic factors of recurrence-free and overall survival in 52 patients with adrenocortical carcinoma
}

\author{
Karolina M. Nowak', Radoslaw Samsel ${ }^{2}$, Andrzej Cichocki ${ }^{2}$, Katarzyna Roszkowska-Purska ${ }^{3}$, Lucyna Papierska \\ ${ }^{1}$ Department of Endocrinology, Centre of Postgraduate Medical Education, Warsaw, Poland \\ ${ }^{2}$ Department of Surgery Clinic of Oncology Memorial M. Sklodowska-Curie Cancer Institute, Warsaw, Poland \\ ${ }^{3}$ Department of Pathology Memorial M. Sklodowska-Curie Cancer Institute, Warsaw, Poland
}

e-mail: knowak@ippt.pan.pl

\section{Background}

Adrenocortical carcinoma (ACC) is a rare and very aggressive tumor with poor prognosis. Its prevalence is estimated on $0.5-2$ cases per million population per year[1]. The overall 5-year survival is generally between $16-44 \%$ [2]. Prognosis is dependent on stage at presentation. The 5-year overall survival rate was $80 \%$ for stage I, $61 \%$ for stage II, $50 \%$ for stage III and $13 \%$ for stage IV disease[3]. The median overall survival is $32-34$ months $[1,4]$. The median recurrence free survival was 42 months[5]. There are several features that may be useful in outcome prediction such as margin status, distant metastasis, tumor invasion of vessels, tumor capsule or adjacent organs, tumor necrosis and mitotic rate $>5 / 50$ high power fields(HPF)[4]. Older age and cortisolsecreting ACCs was also reported to be assocciated with poor survival [6]. According to European Society for Medical Oncology „low-risk” patients with complete resection R0, stage I-II and Ki67 $<10 \%$ have ower risk of disease recurrence compared to patients with R0 together with stage III disease or Ki67>10\% ("high-risk") [7].

\section{Objectives}

The present study has two main objectives:

1) to assess overall survival (OS) and recurrencefree survival (RFS)

2) to identify prognostic factors of OS and predictive features of RFS

\section{Methods}

52 patients were diagnosed with ACC between 2002 -2015.50 of them had primary tumors. Two females suffered from recurrent disease and were excluded from the further analysis (both had survival of 142 and 165 months). We investigated a number of clinical and histological factors such as: age, sex, stage, hormonal activity, tumor size, margin status, thrombus in vena cava, tumor necrosis, tumor invasion of vessels, neighboring structure or adjacent organs, tumor infiltration of capsule with crossing its border, mitotic rate $>20 / 50 \mathrm{HPF}, \mathrm{Ki67}$ score, high-risk patients. Survival curves were calculated using Kaplan-Meier method and the difference between groups or factors were compared using the log-rank test. The univariate and multivariate Cox proportional hazard regression model was performed. Patients with stage I and II were analyzed together (only 3 patients had stage I). Recurrent disease was defined as a new lesion confirmed in imaging.

\begin{tabular}{|c|c|c|c|c|c|c|}
\hline \multirow[t]{2}{*}{ Variable } & \multicolumn{3}{|c|}{ Univariate (OS) } & \multicolumn{3}{|c|}{ Univariate (RFS) } \\
\hline & HR & $95 \% \mathrm{Cl}$ & Pvalue & HR & $95 \% \mathrm{Cl}$ & Pvalue \\
\hline Age $>=50 \mathrm{yrs}$ & 1.03 & $1.0-1.04$ & 0.001 & 1.02 & $1.0-1.03$ & 0.017 \\
\hline Sex ${ }^{* *}$ & 2.57 & $1.21-5.45$ & 0.013 & 2.69 & $1.21-5.99$ & 0.015 \\
\hline Tumor stage & & & & & & \\
\hline I+II & 1 & & & 1 & & \\
\hline IIII & 2.53 & $1.11-5.77$ & 0.026 & 1.93 & $0.83-4.49$ & 0.124 \\
\hline IV & 5.11 & $1.83-14.3$ & 0.002 & 3.7 & $1.2-11.27$ & 0.021 \\
\hline $\begin{array}{c}\text { Invasion in } \\
\text { vessels }\end{array}$ & 4.79 & $1.07-21.3$ & 0.04 & 5.4 & $1.21-24.5$ & 0.027 \\
\hline $\begin{array}{c}\text { Invasion in } \\
\text { neighboring } \\
\text { structure }\end{array}$ & 2.26 & $1.06-4.8$ & 0.033 & 2.16 & $0.98-4.7$ & 0.055 \\
\hline $\begin{array}{l}\text { Infiltration in } \\
\text { adjacent } \\
\text { organs }\end{array}$ & 3.72 & $1.66-8.35$ & 0.001 & 3.78 & $1.55-9.2$ & 0.003 \\
\hline $\begin{array}{l}\text { Mitotic rate } \\
>20 / 50 \mathrm{HPF}\end{array}$ & 6.35 & $1.62-24.8$ & 0.008 & 5.68 & $1.4-21.9$ & 0.012 \\
\hline
\end{tabular}

Table 2. Prognostic factors for overall survival. **female sex was a reference category 2016

Characteristic of patients Female (F) Male (M)

Age (yr) $<50$ years

umor size (cm)

Range (Median)

$<10 \mathrm{~cm}$

$>=10 \mathrm{~cm}$

Stage II

Stage II

Stage IV

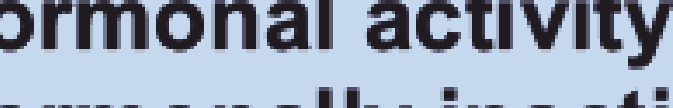

ormonally inactive

Resection status - R0

Tumor necrosis $(n=45)$

Present

Absent

Anvasion in vessels $(n=45)$

Present

Capsular invasion $(n=45)$

Present

Absent

nvasion in neighboring

stracture $(n=45)$

Present

vasion in adjacent organ

$(n=45)$

Present

Mitotic rate $(n=45)$

$<20 / 50 \mathrm{HPF}$

$>=20 / \mathrm{HPF}$

Ki67\% (n=45) $<10 \%$

High-risk

hrombus in vena cava

( $\mathrm{n}=45$ )

Present

\section{Results}

\begin{tabular}{|c|c|c|}
\hline 50 & $\begin{array}{c}5 \text {-year } \\
\text { OS }\end{array}$ & $\begin{array}{c}2 \text {-year } \\
\text { RFS }\end{array}$ \\
\hline $34(68 \%)$ & $44.5 \%$ & $72 \%$ \\
$16(32 \%)$ & $24.8 \%$ & $25 \%$ \\
\hline $24(48 \%)$ & $60 \%$ & $71.6 \%$ \\
$26(52 \%)$ & $14 \%$ & $44 \%$ \\
\hline & & \\
& &
\end{tabular}

\begin{tabular}{|c|c|c|}
\hline $28(56 \%)$ & $42 \%$ & $54 \%$ \\
\hline $3(6 \%)$ & $66.7 \%$ & $100 \%$ \\
\hline
\end{tabular}

\begin{tabular}{|c|c|c|}
\hline $3(6 \%)$ & $66.7 \%$ & $100 \%$ \\
$18(36 \%)$ & $60 \%$ & $77 \%$ \\
\hline
\end{tabular}

\begin{tabular}{l|l|l|}
$20(40 \%)$ & $25 \%$ & $46 \%$ \\
\hline
\end{tabular}

\begin{tabular}{|c|c|c|}
$9(18 \%)$ & $0 \%$ & $18 \%$ \\
\hline $27(54 \%)$ & $34 \%$ & $50 \%$
\end{tabular}

$27(54 \%)$

\begin{tabular}{|l|l|l|}
$12(24 \%)$ & $46 \%$ & $62 \%$ \\
\hline $37(74 \%)$ & $44 \%$ & $64 \%$
\end{tabular}

\begin{tabular}{|l|l|l|}
\hline $3(6 \%)$ & $66 \%$ & $64 \%$ \\
\hline $5(10 \%)$ & $67 \%$ & $30 \%$
\end{tabular}

\begin{tabular}{|l|l|l|}
$5(10 \%)$ & $0 \%$ & $30 \%$ \\
\hline
\end{tabular}

\begin{tabular}{|c|c|c|}
$5(10 \%)$ & $0 \%$ & -- \\
\hline $29(64 \%)$ & $40 \%$ & $56 \%$ \\
\hline
\end{tabular}

\begin{tabular}{|c|c|c|}
\hline $6(13 \%)$ & $75 \%$ & $100 \%$ \\
\hline
\end{tabular}

\begin{tabular}{|l|l|l|}
\hline $21(47 \%)$ & $24 \%$ & $47 \%$ \\
$7(15.5 \%)$ & $71 \%$ & $86 \%$ \\
\hline
\end{tabular}

\begin{tabular}{|l|l|l}
\hline $19(42 \%)$ & $35 \%$ & $45 \%$ \\
\hline
\end{tabular}

\begin{tabular}{|c|c|c|}
\hline $\begin{array}{l}18(40 \%) \\
27(60 \%)\end{array}$ & $\begin{array}{l}22 \% \\
52 \%\end{array}$ & $\begin{array}{l}33 \% \\
78 \%\end{array}$ \\
\hline
\end{tabular}

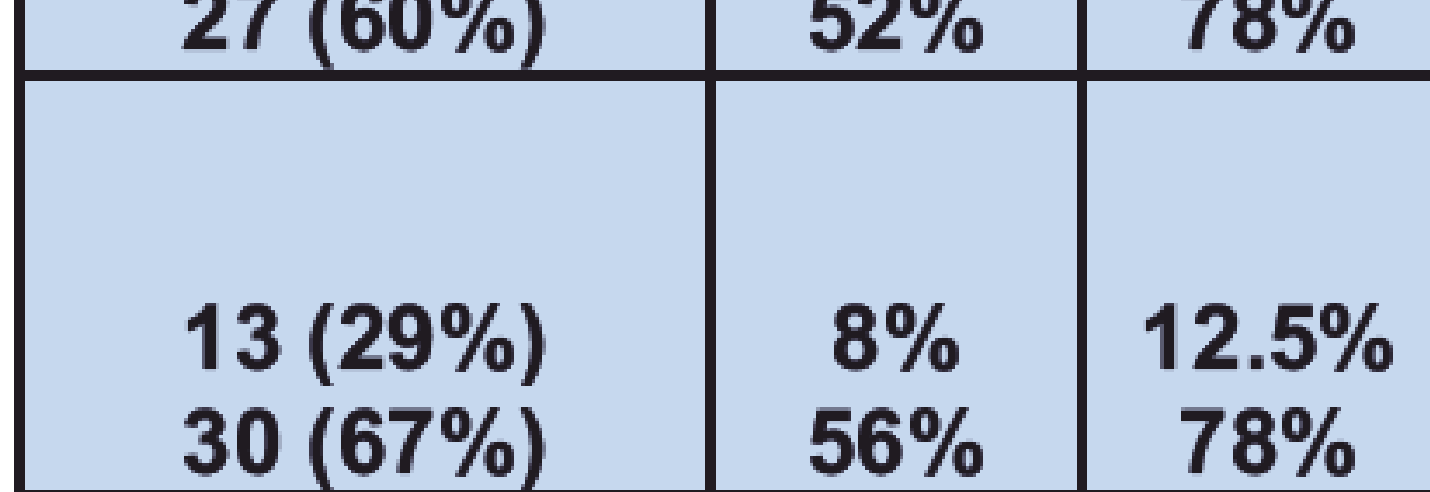

$30(67 \%)$

\begin{tabular}{|c|c|c|}
\hline $12(27 \%)$ & $70 \%$ & $92 \%$ \\
$12(27 \%)$ & $30 \%$ & $24 \%$ \\
\hline $9(18 \%)$ & $52 \%$ & $76 \%$ \\
$9(18 \%)$ & -- & $53 \%$ \\
\hline $16(14 \%)$ & $39 \%$ & $61 \%$ \\
$7(14 \%)$ & $75 \%$ & $83 \%$ \\
\hline & & \\
$5(11 \%)$ & $27 \%$ & $75 \%$ \\
\hline
\end{tabular}

\begin{tabular}{l|l|l|}
$38(84 \%)$ & $43 \%$ & $62 \%$ \\
\hline
\end{tabular}

int's characteristics and difference between groups in 5-year overall survival (OS) rates. High-risk and low-risk patients definition in text.
$16(32 \%) \quad 35 \% \quad 69 \%$

\begin{tabular}{|l|l|l|}
\hline $22(49 \%)$ & $45 \%$ & $70 \%$ \\
\hline
\end{tabular}

\section{Results}

Patients baseline characteristics is provided in table 1 . Results are presented in Table2. The study included 36 females and 16 males of median age 47.5 (22-73) and 57 years (33-77) respectively. $64 \%$ of tumors were hormonally active mainly with overproduction of cortisol (88\%). 44 patients received adjuvant mitotane. 27 patients suffered from recurrent disease. The median of recurrence-free survival was 20.7 months (1.57-132.8) and 25.9 months (2.4-133.4) for overall survival. The median of RFS without stage IV disease was 27 months (2-132.8). Patients $\geq 50$ years had more stage III and IV disease.

Prognostic factors associated with decreased OS :

- in univariate analysis: male sex, age $\geq 50 y e a r s$ disease stage tumor invasion in vessels, neighboring structure or adjacen organs and mitotic rate $>20 / 50 \mathrm{HPF}$.

in multivariate analysis with age as covariate: stage, mitotic rate $>20 / 50 \mathrm{HPF}$ and invasion of neighboring structure or adjacent organs.

Predictive features associated with decreased RFS

in univariate analysis: male sex, age $\geq 50$ years, stage IV, tumo invasion in vessels, infiltration in neighboring structure adjacent organs and mitotic rate $>20 / 50 \mathrm{HPF}$.

-in multivariate analysis with age as covariate: stage IV, mitotic rate $>20 / 50 \mathrm{HPF}$ and invasion of neighboring structure or adjacent organs.

\section{-interestingly, ther}

When the analysis was performed without stage IV disease margin status (R2vsR0) was associated with decresed RFS $(\mathrm{p}=0.01)$

Resection status R0vsR2, „high-risk” factor and tumor necrosis despite the noticeable difference in 5-year OS and 2-year RFS did not reach a statistical significance in Cox model probably due to a small number of events.

Hormonal activity, tumor size, thrombus in vena cava, Ki67 score, tumor infiltration of capsule had no influence on neither OS nor RFS.

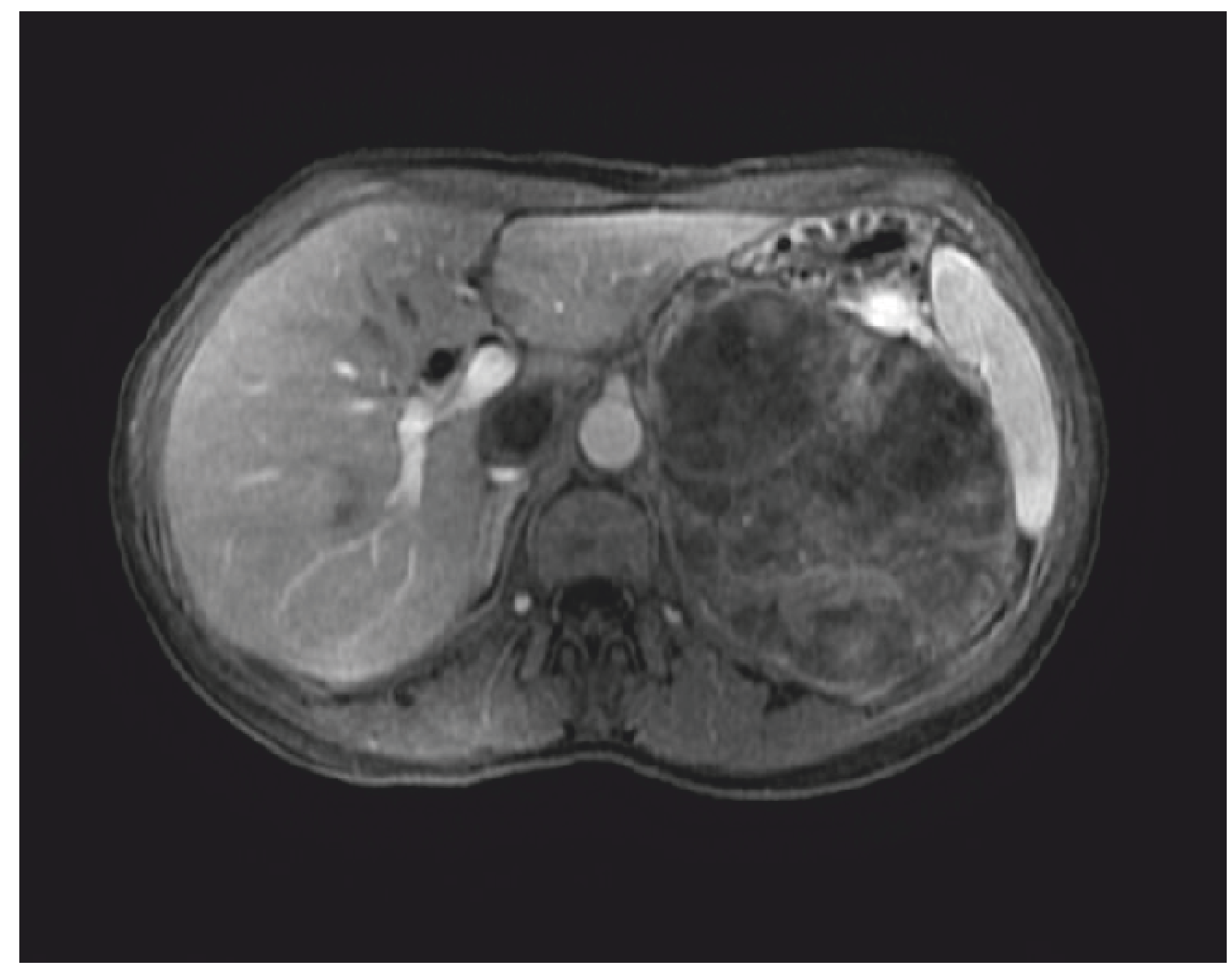

Fig1. Adrenocortical carcinoma

\section{Conclusions}

Our study indicates a major role of prognostic factors on survival in patients with ACC. The 5 -yea survival, median of RFS and OS were worse than previously reported. We identifed a number of features associated with decreased with both OS and RFS such as: male sex, age $\geq 50 y$ years, stage, tumor invasion in vessels, neighboring structure or adjacent organs and mitotic rate $>20 / 50 H P F$. Margin status and tumor necrosis did not reach a significance probably due to a small number of events despite the differences in survival rates. There was no difference in RFS between stage I+II vs stage III disease. Due to the aggressive behavior of ACC and high percentage of relapse it is crucial to conduct more studies in order to help improving survival.

\section{References}

1. Wannis KN, Kanthan R. Diagnostic and prognostic features

. World J Surg Oncol 2015 Mar 24; 13:117

2. Fassnacht $M$, Johanssen $S$ et al. Improved survival in patients with stage

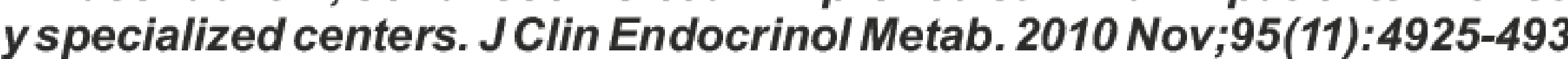
classification for adrenocortical carcinoma: Proposal for a revised TNM classification. Cancer 2009;115:243-250

4. Stojadinovic A, Ghoss

Terzolo $M$, 20:941.

; $356(23): 2372-80$. cortisol-secreting tumors in a series of 202 consecutive patients. J Clin Endocrinol Metab 2006; 91:2650.

A. Berruti, E. Baudin, H. Gelderblom. Adrenal cancer: ESMO Clinical Practice Guide
Clinical practice guidelines. Annals of Oncology 23 (Supplement 7): vii131-vii138, 2012 